\title{
A Case Report, An Adult with Acute Megakaryocytic Leukemia, Immunophenotyping Profile
}

\author{
I G. A. A. Eka Putri Sunari ${ }^{\mathrm{a}}$, Arifoel Hajat ${ }^{\mathrm{b}}$ \\ asiputrisunari@yahoo.com \\ ${ }^{\mathrm{a}}$ Clinical Pathology Specialization Programme, Department of Clinical Pathology, Faculty of Medicine-Dr. \\ Soetomo Teaching Hospital, Universitas Airlangga, Mayjen Prof. Dr. Moestopo street no. 6-8 Surabaya, 60286, \\ Indonesia \\ ${ }^{b}$ Department of Clinical Pathology, Faculty of Medicine-Dr. Soetomo Teaching Hospital, Universitas Airlangga, \\ Mayjen Prof. Dr. Moestopo street no. 6-8 Surabaya, 60286, Indonesia
}

\begin{abstract}
Background: Acute Megakaryocytic Leukemia (AML-M7) is a subtype with 1\% incidence of all adult AML cases. Variation morphology and limitations of immunophenotyping often make AML-M7 difficult to diagnose. The diagnosis of AML-M7 requires morphology and immunophenotyping. This case will discuss about immunophenotyping profile in establishing AMLM7 in an adult patient.

Case Description: A 32-year-old-male, presented with fever, dizziness, loss of appetite and weakness on extremities, signs of bleeding were not found. Physical examination showed conjunctival anemia and decrease of power extremities. Laboratory results showed $\mathrm{Hb} 5.3 \mathrm{~g} / \mathrm{dL}$, WBC 79,700/ $\mu \mathrm{L}$, PLT 9,000/ $\mu \mathrm{L}$, IPF 37.3\%. Blood smear evaluation and bone marrow aspiration resulted in dry tap but obtained formation resembling abnormal micro megakaryocytes and lymphoid cells. Immunophenotyping results were positive on megakaryocytes specific antigen CD 61 and CD36, as well as myeloid markers CD33, CD13, and CD117. Negative results of lymphoid markers were CD3 and CD79a. Patient did not receive chemotherapy and refused treatment. Two days after the patient passed away.

Conclusion : AML-M7 cases in adult patients are very rare. Morphologies are very varied. In this case, morphology of blasts was difficult to distinguish with lymphoid cells because cytoplasm bleps were found. Immunophenotyping results showed positive CD61 and CD36 and negative lymphoid markers (CD3, CD79a) according to AML M7. Myeloid markers were positive in this case. Thus, it is concluded that this case was AML-M7.
\end{abstract}

Keywords: Megakaryocytic leukemia; hematology malignancy; immunophenotyping profile

\section{Introduction}

Acute myeloid leukemia is a hematology malignancy characterized by excessive clonal proliferation of myeloid precursors and failure of differentiation. Proliferation that is not accompanied by differentiation results in the unavailability of non-functioning immature cells (1). Based on the French-British-American classification, AML is divided into M0-M7 based on morphology and cytochemistry (2).

AML-M7 is a rare subtype of acute myeloid leukemia. In adult patients, the prevalence is around $1 \%$. The diagnosis of AML M7 according to the French-American-British Cooperative Group (FAB) Criteria was made using bone marrow aspirate which showed megakaryoblastic/ megakaryocytic cell lines $>30 \%$ of all cells. Megakaryocytic cell lines were positive for clear using electron microscopy and reacted to platelet peroxidase or using monoclonal or polyclonal specific antibodies. However, myelofibrosis due to increased reticulin is common in patients with AML M7, so that adequate sampling of bone marrow is difficult to obtain. Blast counts on bone marrow aspirates can be incorrect. Counting the number of blasts can be done if sufficient bone marrow biopsy results are obtained (3).

Morphological examination is generally carried out using Wright's stain on a light microscope. Megakaryoblasts vary in diameter from the size of a small lymphocyte to three times their size. Megakaryoblasts 
are generally medium to large (12-18 pm) with rounded, slightly irregular, or indented nuclei with fine reticular chromatin and 1-3 nucleoli. The cytoplasm is basophilic, often agranular, and may show formation of blebs or pseudopodia. In some cases, blasts are mostly small resembling lymphoblasts (4).

Megakaryoblasts express the platelet glycoproteins CD41 (glycoprotein IIb), CD42b (glycoprotein Ib) and CD61 (glycoprotein IIIa). Expression of CD41 or CD61 from the cytoplasm was more specific and sensitive than cell surface staining, along with the possibility of platelet attachment to the blast (satelliteism) leading to false positive interpretations on flowcytometry $(5,6)$.

\section{Case Presentation}

A man, 32 years old, came with a fever. The patient had fever since 2 days before admission to the hospital. Complaints accompanied by dizziness, weakness and decreased appetite. Two weeks ago the patient received a transfusion of $4 \mathrm{PRC}$ bags due to $\mathrm{Hb} 6.3$ and a suspected haematological malignancy.

On physical examination, the patient was pale and there was no swollen lymph nodes. No bleeding under the skin. Chest examination was within normal limits. The liver and spleen were not palpable. Complete blood count results showed $\mathrm{Hb} 5.3 \mathrm{~g} / \mathrm{dL}$, WBC $79.710^{3} / \mathrm{uL}$ (neutrophils $66.8 \%$, lymphocytes 13.3\%, monocytes 19.3), Platelets $910^{3} / \mathrm{uL}$, IPF $37.3 \%$. The results of the peripheral blood cell picture showed normochromic anisopoikilocytosis anemia, leukocytosis with abnormal micromegakaryocytes $26 \%$, immature granulocytes $(+)$ and thrombocytopenia (figure 1).

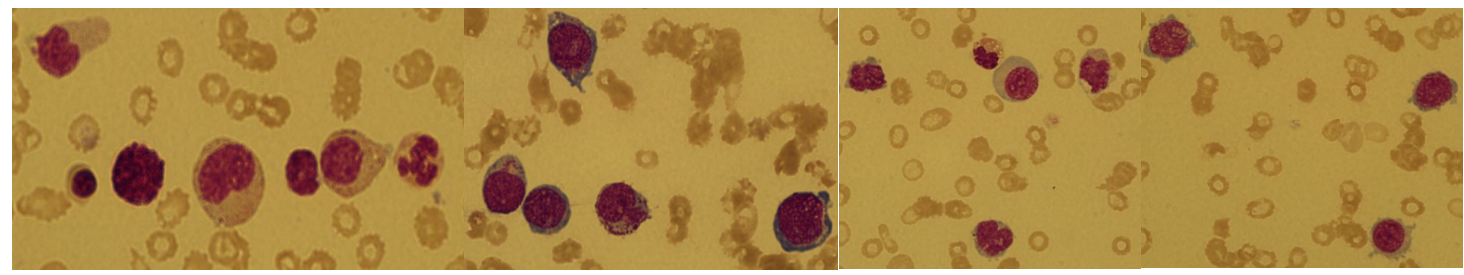

Figure 1. Abnormal morphology of micromegakaryocytes in peripheral blood picture

Bone marrow aspiration (BMA) obtained dry tap and no fragments. The formation of immature mononuclear cells with cytoplasmic bleps was found which was suspected as abnormal micromegakaryocytes in BMA (figure 2).

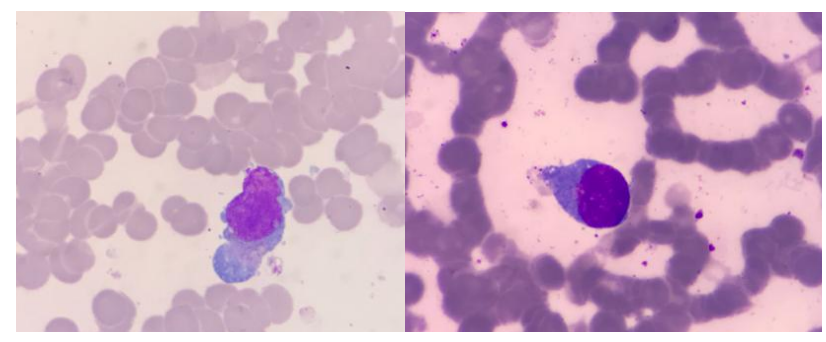

Figure 2. Abnormal morphology of micromegakaryocytes in bone marrow aspiration

Peripheral blood immunophenotyping was positive for CD 33, CD 36, CD 117 and CD 61 (table 2). CD 33 and CD117 were markers for myeloid, CD 61 was megakaryocyte-specific antigen. Clinical chemistry examination found an increase in SGOT 117, SGPT 882, uric acid 19.8. Non-reactive HBsAg and anti-HCV immunological examination. The patient was not subjected to bone radiology examination to confirm fibrosis. 
Table 1. Immunophenotyping result

\begin{tabular}{cc}
\hline Marker & Result \\
\hline CD 33 & Positive \\
CD 34 & Negative \\
CD 36 & Positive \\
CD 117 & Positive \\
CD 3 & Negative \\
CD 13 & Positive (Dim) \\
CD 61 & Positive \\
CD 79a & Negative \\
MPO & Negative \\
\hline
\end{tabular}

The patient was suspected as AML M7 by the clinician and received supportive therapy. Treatment to the patient was stopped because the patient asked to be forced to go home before chemotherapy started. Two days after returning home, patient died.

\section{Disscusion}

Acute Myeloblastic Leukemia (AML) M7 is a subtype of AML according to the FAB. The diagnosis of AMLM7 was defined as a megakaryoblast count $>50 \%$ of the total blast count $(>20 \%)$ found in peripheral blood picture or bone marrow (5). The routine blood results of this patient showed $\mathrm{Hb} 5.3 \mathrm{~g} / \mathrm{dL}$, WBC $79.700 / \mu \mathrm{L}$, PLT $9000 / \mu \mathrm{L}$, IPF $37.3 \%$. Low haemoglobin and platelets and high leukocytes indicate a leukemia.

In this case, a high value was obtained for the Immature Platelet Fraction (IPF). High IPF values were found in patients with ITP with an IPF range of $>16.39 \%$ (7). In non-ITP patients, a lower IPF value is often obtained, but in this case a high IPF value was obtained but did not support an ITP, so the suspicion of immature platelets could not be ruled out.

The morphological description of megakaryocytes is very pleomorphic with small to large cell shapes, round with little cytoplasm, nuclei hard to find and bleep cytoplasm is often found (5). The peripheral blood picture results in this case showed a small size mononuclear cell with bleep cytoplasm, loose chromatin and indistinct nucleus which suspected an abnormal micromegakaryocyte or lymphoblast. The results of BMA are difficult to evaluate due to dry tap (failure of bone marrow aspiration) so it cannot be determined whether the number of blasts is $>20 \%$ and megakaryoblasts $>50 \%$ of the number of blasts. Dry tap can be caused by bone marrow fibrosis. This condition occurs frequently in patients with AML M7 (8).

In a previous report, there were 7 patients who had moderate and extensive myelofibrosis resulting in dry tap and inaccurate diagnosis of AML-M7 (9). Another study on AML-M7 also found that dry taps suggested a bone marrow biopsy to support the diagnosis (10). In this case, the criteria for blasts $>20 \%$ and megakaryoblasts $>50 \%$ of the number of blasts cannot be used. Immunophenotyping, bone marrow biopsy and immunohistochemistry are needed in establishing the diagnosis. In this case it could not be determined whether myelofibrosis was present or not because a bone marrow biopsy was not performed, but the suspicion of myelofibrosis could not be ruled out.

In this case several markers were tested to narrow the diagnosis. Myeloid lineage was seen at CD33, CD13, CD117, and MPO. Lymphoid lineage was seen on CD 7 and CD 3. The markers for megakaryocytes were assessed at specific $\mathrm{CD} 36$ and $\mathrm{CD}$ 61. The results of the immunophenotyping examination in this case were positive on CD 33, CD 36, CD 117, CD 13 and CD 61. Negative results on CD3, CD 79a, CD 34 and MPO. Immunophenotyping that describes megakaryocytes is the expression of CD 41, CD 61, factor VIII, CD 36, CD 38, CD 7 aberration, negative on myeloid markers including MPO, CD 34 and CD 117. CD61 (6).

The CD 61 marker, also known as GPIIIa, is a glycoprotein found on platelets, monocytes, endothelial cells, smooth muscle, and mast cells. This protein is encoded by the Integrin Beta-3 (ITGB3) gene. CD 61 has a role in platelet aggregation and also as a receptor for fibrinogen and von Willbrand factor. Until now, CD 61 has been used as an immunophenotyping marker to assess megakaryocyte leukemia. The CD 36 marker also known 
as platelet glycoprotein 4 (GPIV) is a protein that is encoded by the CD 36 gene. CD 36 is an integral membrane protein found on the surface of platelets. Together with CD 41 and CD 61, CD 36 is used as an immunophenotyping marker in the diagnosis of AML-M7 (4).

In this case, a negative result for the lymphoid marker CD 3, CD 79a could rule out the presence of lymphoblasts. The suspicion of AML was strengthened by positive immunophenotyping of myeloid lineage markers, namely CD 33, CD 13, CD 117. Positive results on megakaryocyte markers CD 36 and CD 61 further strengthen the diagnosis of AML-M7. Previous studies reported the probability of the appearance of myeloid markers in AML-M7 namely CD33, CD13 and CD117 around 75\%, 30\% and 30\% (6).

\section{Conclusion}

The results of routine blood examination, peripheral blood smear, bone marrow aspiration and immunophenotyping point to AML-M7 so it can be concluded that the diagnosis of this patient is AML-M7.

\section{Acknowledgements}

The author would like to thank Mr. Arifoel Hajat, MD., who has helped and guided the writing of this case report.

\section{References}

1. Stone RM, O’Donnell MR, Sekeres MA. Acute Myeloid Leukemia. Hematology. 2004 Jan 1;2004(1):98-117.

2. Walter RB, Othus M, Burnett AK, Löwenberg B, Kantarjian HM, Ossenkoppele GJ, et al. Significance of FAB subclassification of "acute myeloid leukemia, NOS" in the 2008 WHO classification: analysis of 5848 newly diagnosed patients. Blood. 2013 Mar 28;121(13):2424-31.

3. Bennet JM. Criteria for the Diagnosis of Acute Leukemia of Megakaryocyte Lineage (M7). Annals of Internal Medicine. 1985 Sep 1;103(3):460.

4. Lichtman MA, Kaushansky K, Kipps TJ et al. Williams manual of hematology. 8th ed. The McGrawHill Companies, Inc.; 2011.

5. Naeim, Faramarz; Rao, P; Song, Sophie; Phan R. Atlas of Hematopathology 2nd Edition. 2nd Editio. Elsevier; 2018. 273-276 p.

6. Campana D, Behm FG. Immunophenotyping of leukemia. Journal of Immunological Methods. 2000 Sep;243(1-2):59-75.

7. Naz A, Mukry SN, Shaikh MR, Bukhari AR, Shamsi TS. Importance of immature platelet fraction as predictor of immune thrombocytopenic purpura. Pakistan Journal of Medical Sciences. 2016 May $7 ; 32(3)$.

8. Sharma S, Nangia A, Jain Malhotra S, Narayan S, Harbhajanka A, Singh S. Clinico-Haematological Profile of Acute Megakaryoblastic Leukaemia: Report of Five Cases. Advances in Hematology. 2009;2009:1-4.

9. Zhao G, Wu W, Wang X, Gu J. Clinical diagnosis of adult patients with acute megakaryocytic leukemia. Oncology Letters. 2018 Sep 25;

10. O Olatunji P. A case of acute megakaryoblastic leukaemia (FAB M7), a rare type of acute myeloid leukemia (AML), in a teenager. Malawi Medical Journal. 2018 Dec 31;30(4):298. 